\title{
Aberration Corrected Off-Axis Electron Holography of Layered Transition Metal Dichalcogenides
}

Amir H. Tavabi ${ }^{1}$, Florian Winkler ${ }^{1}$, Yung-Chang Lin $^{2}$, Kazu Suenaga ${ }^{2}$, Emrah Yucelen ${ }^{3}$, Rafal E. Dunin-Borkowski ${ }^{1}$, Beata E. Kardynal ${ }^{4}$

1 Ernst Ruska- Centre for Microscopy and Spectroscopy with Electrons and Peter Grünberg Institute 5, Forschungszentrum Jülich, Germany

2 National Institute of Advanced Industrial Science and Technology (AIST), Tsukuba, Japan

3 FEI Company, Eindhoven, The Netherlands

4 Peter Grünberg Institute 9, Forschungszentrum Jülich, Germany

Two-dimensional transition metal dichalcogenides (TMDs) have attracted great attention for device applications in the last decade, in part due to their direct band gaps [1, 2]. Off-axis electron holography can provide unique information about the properties of TMDs that is complementary to the information provided by more conventional transmission electron microscopy (TEM) techniques.

TEM samples of TMDs were prepared by cleaving, followed by transfer onto an elastomer gel film. Subsequently, the flakes were transferred onto gold-coated holey SiN membranes without the use of wet chemicals. Before TEM measurements, each specimen was annealed at $85^{\circ} \mathrm{C}$ overnight in a vacuum furnace $\left(<1 \times 10^{-5}\right.$ mbar $)$.

Medium and high resolution off-axis electron holograms of a wide range of TMDs with nominal composition $\mathrm{MX}_{2}(\mathrm{M}: \mathrm{Mo}, \mathrm{W}, \mathrm{Re} ; \mathrm{X}: \mathrm{S}, \mathrm{Se}$ ) were acquired at accelerating voltages of 50, 60 and $80 \mathrm{kV}$ using spherical and chromatic aberration corrected TEMs.

Figure 1 shows representative results acquired at $80 \mathrm{kV}$ using off-axis electron holography from an $\mathrm{MoS}_{2}$ flake. The holographic interference fringe spacing was $30 \mathrm{pm}$ and a mask corresponding to a spatial resolution of $90 \mathrm{pm}$ was applied to the sideband (Fig. 1c) before reconstructing the phase image. Figure 1d shows part of a phase image reconstructed from the hologram, showing a two and three monolayer region of the flake and the presence of some contamination at the specimen edge.

Phase images such as that shown in Fig. 1d were used to determine the mean inner potential, the number of monolayers and the number of atoms in each atomic column across the field of view.

Challenges in such measurements were found to arise from the stability of the specimen and the instrument, the presence of residual aberrations, contamination on the specimen surfaces and electronbeam-induced charging and damage of the specimen. Charging effects are visible in the line profile shown in Fig. 1e, in the form of a slight slope in the phase in a region of uniform thickness inside the specimen when compared with the slope of the phase in vacuum. Approaches for overcoming such problems will be discussed.

References:

[1] Radisavljevic, B. et al, Nature Nanotech. 6 (2011), p. 147.

[2] Wang, Q. et al, Nature Nanotech. 7 (2012), p. 699. 

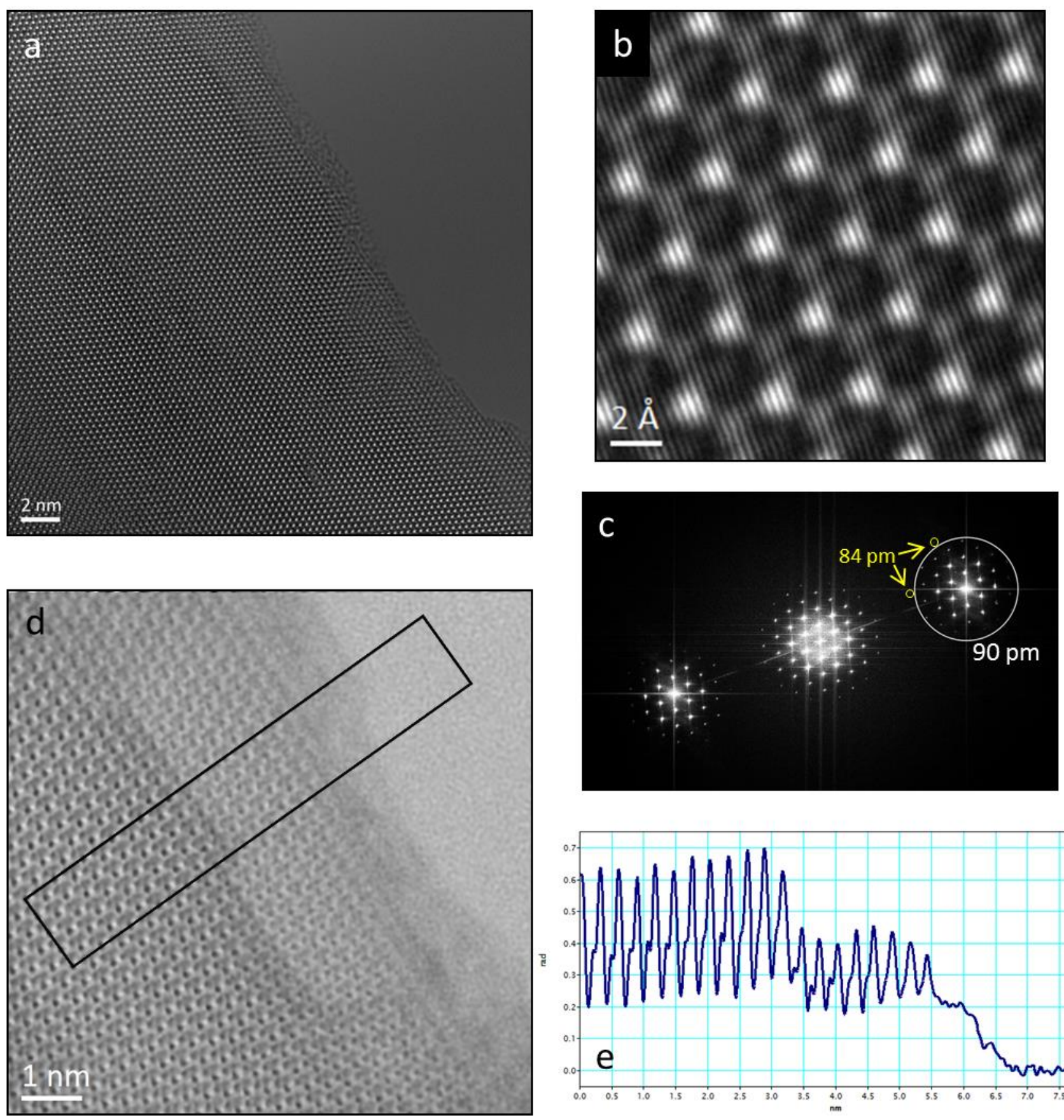

Figure 1. a) Off-axis electron hologram of an $\mathrm{MoS}_{2}$ flake recorded at $80 \mathrm{kV}$. b) Magnified region of electron hologram showing fine shifts of interference fringes in a three-layer flake. c) Fast Fourier transform of the hologram, showing information at $84 \mathrm{pm}$. d) Part of a reconstructed phase image of two and three layer $\mathrm{MoS}_{2}$, showing the presence of contamination at the specimen edge. e) Phase shift profile extracted from the black rectangle marked in the phase image. 